\title{
Extending multidimensional poverty identification: from additive weights to minimal bundles
}

\author{
Sam Jones ${ }^{1}$
}

Received: 28 May 2019 / Accepted: 19 January 2021 / Published online: 17 May 2021

(C) UNU-WIDER 2021, corrected publication 2021

\begin{abstract}
In the popular class of multidimensional poverty measures introduced by Alkire and Foster (2011), a threshold switching function is used to identify who is multidimensionally poor. This paper shows that the weights and cut-off employed in this procedure are generally not unique and that such functions implicitly assume all groups of deprivation indicators of some fixed size are perfect substitutes. To address these limitations, I show how the identification procedure can be extended to incorporate any type of positive switching function, represented by the set of minimal deprivation bundles that define a unit as poor. Furthermore, the Banzhaf power index, uniquely defined from the same set of minimal bundles, constitutes a natural and robust metric of the relative importance of each indicator, from which the adjusted headcount can be estimated. I demonstrate the merit of this approach using data from Mozambique, including a decomposition of the adjusted headcount using a 'one from each dimension' non-threshold function.
\end{abstract}

Keywords Multidimensional poverty $\cdot$ Switching functions $\cdot$ Mozambique

\section{Introduction}

The conceptualization and measurement of well-being as a multidimensional phenomenon is long-standing. Following the seminal contribution of Alkire and Foster (2011a), who proposed a generalization of the Foster-Greer-Thorbecke class of poverty measures (Foster et al. 1984) to multiple domains of deprivation, their approach to measuring multidimensional well-being has been used extensively and further extended (e.g., Bennett and Mitra 2013; Alkire and Santos 2014; Alkire and Seth 2015; Seth and Alkire 2017). A Google Scholar search reveals nearly 1,000 papers applying or discussing their framework. ${ }^{1}$ Since 2010, the UN's annual Human Development Report has included a multidimensional poverty index of the Alkire-Foster type, based on survey data for more than 100 countries

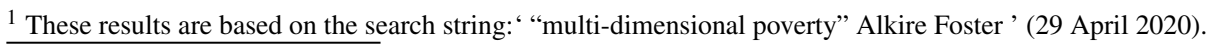

Sam Jones

jones@wider.unu.edu
}

1 UNU-WIDER, Ministry of Economy and Finance, Maputo, Mozambique 
and covering ten dimensions of well-being. And both Mexico and Colombia have adopted this type of multidimensional index to guide and track progress in national poverty reduction (e.g., Angulo et al. (Angulo et al. 2016)).

In line with many approaches to multidimensional poverty measurement, as well as the construction of various composite indexes (e.g., Seth 2009; Foster et al. 2013; Jones 2016), the Alkire-Foster procedure aggregates observations across various separate deprivation indicators by applying a set of weights (Decancq and Lugo 2013). ${ }^{2}$ In addition to these, an overall cut-off (threshold) is applied to determine whether a given weighted sum of realized deprivations is sufficient to identify a unit as poor. As detailed in Section 2, this process of multidimensional poverty identification, or aggregation across deprivations at the unit level, first involves application of a positive threshold switching function, which switches from zero to one when the weighted sum of inputs (binary poverty indicators) exceeds a defined threshold. This binary output is then aggregated across units to yield the poverty headcount. On the face of it, the specific weights and cut-off entering the threshold function play a central role in poverty identification and, in this spirit, have been the focus of many studies of different identification schemes (e.g., Alkire et al. 2011; Santos and Villatoro 2018). ${ }^{3}$

The first objective of this paper is to revisit the nature of identification under the AlkireFoster procedure. In Section 2, I highlight that the weights and cut-offs are generally not unique to each function, in the sense that different sets of weights or cut-offs are often able to produce identical identification results. To give a simple example, assume three dimensions attributed equal weights (1/3). Based on a threshold switching function with cut-off of $1 / 2$, all those deprived in at least two dimensions will be identified as poor. But the same result obtains if the weights are changed to $0.1,0.45,0.45$, even if this vector seems to suggest the first dimension is much less important. ${ }^{4}$ As such, the specific choice of weights does not necessarily provide an accurate guide to the influence of each deprivation indicator in identifying the poor. The reliance on these same numerical weights to calculate the intensity of deprivations at the unit level also implies there is no unique mapping from the identification function to the adjusted poverty headcount. Simple examples and insights from game theory further clarify that only restricted types of identification schemes can be encoded using the Alkire-Foster procedure. Richer and more appealing schemes, such as 'one from each dimension' (where to be poor, a unit must be deprived in at least one indicator within each of various aggregate dimensions), generally cannot be encoded via (single) threshold functions.

My second objective is to propose a more general alternative to multidimensional poverty identification (and subsequent analysis), extending the types of positive switching functions that can be incorporated within an Alkire-Foster form of analysis. In Section 3, I show that the fundamental basis of such an approach is the enumeration of all feasible bundles of deprivations defined from a chosen set of binary deprivation indicators. As per a logical truth table, any positive switching (Boolean) function that maps a given set of realized deprivations to either one or zero (namely, poor or non-poor) defines a complete vector of true points. These true points are unique to each function - they index which bundles of realized deprivations define a unit as poor and can be used directly to calculate a multidimensional

\footnotetext{
${ }^{2}$ Key contributions to the literature on constructing multidimensional measures of well-being include Maasoumi (1986), Atkinson (2003), and Bourguignon and Chakravarty (2003). For a broader discussion see Anand et al. (2011).

3 Throughout I use the term 'identification' to refer to multidimensional identification (aggregation across deprivations at the unit level) and I use 'aggregation' to refer to aggregating (averaging) over units.

4 Thanks to a referee for suggesting this example.
} 
poverty headcount. In addition, I show that any chosen switching function can be uniquely represented by its associated collection of minimal positive deprivation bundles, being the smallest bundles that define a unit as poor. ${ }^{5}$ These bundles are important since they also form a unique basis for the calculation of the relative importance of each indicator, namely the Banzhaf power index, which can be used to derive the adjusted headcount and pursue associated analysis. Lastly, Section 4 draws on data from Mozambique to demonstrate how this more general procedure works in practice, including how a 'one from each dimension' function can be easily deployed.

\section{Identification via weights and a cut-off}

This section reflects on two limitations of what might be called the 'weights and cut-off' approach to poverty identification. To begin, some basic definitions are in order. Following the conventional Alkire-Foster set-up, assume there exists an $n \times m$ matrix of binary deprivation indicators with $n$ units of interest (e.g., individuals or households) and $m$ indicators of deprivation. The elements of this matrix, denoted $D$, are labelled $d_{i j}$ such that $d_{i j}=1$ if unit $i \in\{1, \ldots, n\}$ is deprived in indicator $j \in\{1, \ldots, m\}$ and zero otherwise. ${ }^{6}$ To identify the poor, a normalized vector of weights $\vec{w}=\left(w_{1}, w_{2}, \ldots, w_{m}\right)$ and single cut-off $(k)$ is required. Unit $i$ 's poverty status is then ascertained from a positive threshold switching function: ${ }^{7}$

$$
h_{i}=1\left[\sum_{j=1}^{m} d_{i j} w_{j} \geq k\right]
$$

where $\forall j: 0<w_{j}<1, \sum_{j=1}^{m} w_{j}=1,0<k \leq 1$

which switches from zero to one when the sum of weights in which the unit is deprived exceeds the chosen threshold. A particular combination $(\vec{w}, k)$ therefore corresponds to a specific poverty identification function; and we can say a true point of that function obtains when it yields a positive output for a given input vector. ${ }^{8}$ Following this identification step, the aggregate multidimensional poverty headcount is obtained from the sample mean of the resultant vector of unit-specific poverty indicators: $H(D ; \vec{w}, k)=n^{-1} \sum_{i=1}^{n} h_{i}$. The adjusted headcount, which takes into account differences in the intensity of poverty among the poor, is derived as: $M_{0}(D ; \vec{w}, k)=n^{-1} \sum_{i=1}^{n} h_{i}\left(\sum_{j=1}^{m} d_{i j} w_{j}\right)$ such that: $H \geq M_{0} .^{9}$

\footnotetext{
${ }^{5}$ Hereafter, all bundles are assumed to be positive in the sense that no indicator enters in complemented form.

${ }^{6}$ I presume throughout that both the deprivation indicators and unit-wise observations are taken as given. Note the binary deprivation measures can be obtained in a variety of ways, including via transformations of continuous measures.

7 A switching (Boolean) threshold function in $m$ inputs assigns to each binary sequence of length $m$ the value one if some combination of inputs exceeds a given threshold and zero otherwise. A positive threshold function is a threshold function that is positive in all its inputs, meaning that for any sequence of $m$ binary inputs, turning one input from one to zero cannot increase the functions' output value.

${ }^{8}$ As elaborated further below, one can classify all unique feasible groups of inputs as belonging to either the set of false points or the set of true points, denoting $\mathcal{T}(h)$ as the collection of all true input vectors defined by function $h$.

${ }^{9}$ Sample weights can be applied in the averaging stage, but are not shown here for simplicity of presentation. Further discussion of aggregation over units (e.g., households) is not pursued in this paper.
} 


\subsection{Non-uniqueness}

Despite the fundamental roles of the weight vector and cutoff for poverty identification, an overlooked feature of the Alkire-Foster approach is that what may appear to be distinct choices for $(\vec{w}, k)$ can often map to identical poverty identification results for the same matrix of observed deprivations - i.e., different choices of $(\vec{w}, k)$ typically do not equate to unique poverty definitions. This non-uniqueness property is illustrated in Table 1 , which simulates a case of five deprivation indicators, with each row describing a particular choice for $(\vec{w}, k)$. The first five columns indicate the weights ascribed to each indicator, and the sixth column gives the cut-off. The last two columns report the headcount $(H)$ and adjusted headcount $\left(M_{0}\right)$ for each identification function, calculated using survey data from Mozambique (see Section 4 for more details).

Row 1(a) is a naïve scheme that ascribes equal weights to each indicator; so, with $k=0.8$, a unit (household) must be deprived in at least four indicators to be considered poor. Rows 1(b) and 1(c) present alternative weights and cut-offs (generated using pseudorandom numbers); but both of these functions map to exactly the same poverty definition as 1(a) - i.e., the sum of any four of the weights (and no less) is always required to breach the associated cut-off. Rows 2(a)-(c) of Table 1 provide a second example of different choices of $(\vec{w}, k)$ that map to equivalent poverty definitions. The combinations of weights that are sufficient to exceed the cut-off are more varied here, ranging from four to just two. In keeping with the first example, the rank order of the weights is not stable across the rows; also, the relative magnitude of weights varies considerably. For instance, in Row 2(a) the ratio $w_{1} / w_{5}$ is greater than three; but in Row 2(c), the same ratio is less than one. Despite these differences, each of the three row vectors identifies the same units as either poor or non-poor; and the headcount measure is thereby identical.

The mechanics behind this result, which prefigure results to come and are not mere statistical flukes, are demonstrated in Table 2 . Here I enumerate all $2^{5}=32$ unique combinations of deprivations in five indicators, which represents the basis for a logical truth table. For each unique combination of deprivations, shown in the rows, I apply the (Alkire-Foster) threshold switching function based on the different values of $(\vec{w}, k)$ from rows $1(\mathrm{a})-1$ (c) and

Table 1 Examples of equivalent poverty identification functions

\begin{tabular}{|c|c|c|c|c|c|c|c|c|}
\hline & $w_{1}$ & $w_{2}$ & $w_{3}$ & $w_{4}$ & $w_{5}$ & $k$ & $H$ & $M_{0}$ \\
\hline 1(a) & 0.200 & 0.200 & 0.200 & 0.200 & 0.200 & 0.800 & 0.346 & 0.307 \\
\hline 1(b) & 0.179 & 0.156 & 0.222 & 0.235 & 0.207 & 0.684 & 0.346 & 0.303 \\
\hline $1(\mathrm{c})$ & 0.165 & 0.221 & 0.184 & 0.211 & 0.220 & 0.673 & 0.346 & 0.307 \\
\hline c.v. & 0.097 & 0.171 & 0.096 & 0.084 & 0.048 & 0.098 & 0.000 & 0.007 \\
\hline 2(a) & 0.328 & 0.047 & 0.459 & 0.068 & 0.099 & 0.515 & 0.490 & 0.422 \\
\hline 2(b) & 0.300 & 0.027 & 0.483 & 0.050 & 0.140 & 0.514 & 0.490 & 0.427 \\
\hline 2(c) & 0.136 & 0.114 & 0.409 & 0.148 & 0.194 & 0.536 & 0.490 & 0.398 \\
\hline c.v. & 0.408 & 0.724 & 0.083 & 0.588 & 0.331 & 0.024 & 0.000 & 0.036 \\
\hline
\end{tabular}

Note: Each row sets out the parameters of an Alkire-Foster poverty identification function in five indicators, containing a set of weights $(\vec{w})$ and cut-off $(k)$; columns $H$ and $M_{0}$ respectively report the aggregate headcount and adjusted headcount, calculated by applying these parameters to data from Mozambique in 2014/15 (see Section 4); c.v. denotes coefficient of variation

Source: own calculations 
Table 2 Enumeration of all deprivation bundles in five indicators, with true points of selected identification functions

\begin{tabular}{|c|c|c|c|c|c|c|c|c|c|c|c|c|c|}
\hline \multirow[b]{2}{*}{ id. } & \multicolumn{5}{|c|}{ Bundles } & \multirow[b]{2}{*}{$\pi$} & \multicolumn{7}{|c|}{ True points of different identification functions } \\
\hline & $d_{1}$ & $d_{2}$ & $d_{3}$ & $d_{4}$ & $d_{5}$ & & 1(a) & $1(b)$ & $1(\mathrm{c})$ & 2(a) & $2(b)$ & $2(\mathrm{c})$ & 3 \\
\hline 1 & 0 & 0 & 0 & 0 & 0 & 0.089 & 0 & 0 & 0 & 0 & 0 & 0 & 0 \\
\hline 2 & 1 & 0 & 0 & 0 & 0 & 0.082 & 0 & 0 & 0 & 0 & 0 & 0 & 0 \\
\hline 3 & 0 & 1 & 0 & 0 & 0 & 0.021 & 0 & 0 & 0 & 0 & 0 & 0 & 0 \\
\hline 4 & 1 & 1 & 0 & 0 & 0 & 0.125 & 0 & 0 & 0 & 0 & 0 & 0 & 0 \\
\hline 5 & 0 & 0 & 1 & 0 & 0 & 0.107 & 0 & 0 & 0 & 0 & 0 & 0 & 0 \\
\hline 6 & 1 & 0 & 1 & 0 & 0 & 0.048 & 0 & 0 & 0 & 1 & 1 & 1 & 1 \\
\hline 7 & 0 & 1 & 1 & 0 & 0 & 0.006 & 0 & 0 & 0 & 0 & 0 & 0 & 1 \\
\hline 8 & 1 & 1 & 1 & 0 & 0 & 0.024 & 0 & 0 & 0 & 1 & 1 & 1 & 1 \\
\hline 9 & 0 & 0 & 0 & 1 & 0 & 0.000 & 0 & 0 & 0 & 0 & 0 & 0 & 0 \\
\hline 10 & 1 & 0 & 0 & 1 & 0 & 0.000 & 0 & 0 & 0 & 0 & 0 & 0 & 1 \\
\hline 11 & 0 & 1 & 0 & 1 & 0 & 0.000 & 0 & 0 & 0 & 0 & 0 & 0 & 1 \\
\hline 12 & 1 & 1 & 0 & 1 & 0 & 0.000 & 0 & 0 & 0 & 0 & 0 & 0 & 1 \\
\hline 13 & 0 & 0 & 1 & 1 & 0 & 0.000 & 0 & 0 & 0 & 1 & 1 & 1 & 0 \\
\hline 14 & 1 & 0 & 1 & 1 & 0 & 0.000 & 0 & 0 & 0 & 1 & 1 & 1 & 1 \\
\hline 15 & 0 & 1 & 1 & 1 & 0 & 0.000 & 0 & 0 & 0 & 1 & 1 & 1 & 1 \\
\hline 16 & 1 & 1 & 1 & 1 & 0 & 0.000 & 1 & 1 & 1 & 1 & 1 & 1 & 1 \\
\hline 17 & 0 & 0 & 0 & 0 & 1 & 0.001 & 0 & 0 & 0 & 0 & 0 & 0 & 0 \\
\hline 18 & 1 & 0 & 0 & 0 & 1 & 0.008 & 0 & 0 & 0 & 0 & 0 & 0 & 1 \\
\hline 19 & 0 & 1 & 0 & 0 & 1 & 0.006 & 0 & 0 & 0 & 0 & 0 & 0 & 1 \\
\hline 20 & 1 & 1 & 0 & 0 & 1 & 0.053 & 0 & 0 & 0 & 0 & 0 & 0 & 1 \\
\hline 21 & 0 & 0 & 1 & 0 & 1 & 0.017 & 0 & 0 & 0 & 1 & 1 & 1 & 0 \\
\hline 22 & 1 & 0 & 1 & 0 & 1 & 0.039 & 0 & 0 & 0 & 1 & 1 & 1 & 1 \\
\hline 23 & 0 & 1 & 1 & 0 & 1 & 0.012 & 0 & 0 & 0 & 1 & 1 & 1 & 1 \\
\hline 24 & 1 & 1 & 1 & 0 & 1 & 0.115 & 1 & 1 & 1 & 1 & 1 & 1 & 1 \\
\hline 25 & 0 & 0 & 0 & 1 & 1 & 0.000 & 0 & 0 & 0 & 0 & 0 & 0 & 0 \\
\hline 26 & 1 & 0 & 0 & 1 & 1 & 0.007 & 0 & 0 & 0 & 0 & 0 & 0 & 1 \\
\hline 27 & 0 & 1 & 0 & 1 & 1 & 0.003 & 0 & 0 & 0 & 0 & 0 & 0 & 1 \\
\hline 28 & 1 & 1 & 0 & 1 & 1 & 0.047 & 1 & 1 & 1 & 1 & 1 & 1 & 1 \\
\hline 29 & 0 & 0 & 1 & 1 & 1 & 0.005 & 0 & 0 & 0 & 1 & 1 & 1 & 0 \\
\hline 30 & 1 & 0 & 1 & 1 & 1 & 0.017 & 1 & 1 & 1 & 1 & 1 & 1 & 1 \\
\hline 31 & 0 & 1 & 1 & 1 & 1 & 0.016 & 1 & 1 & 1 & 1 & 1 & 1 & 1 \\
\hline 32 & 1 & 1 & 1 & 1 & 1 & 0.151 & 1 & 1 & 1 & 1 & 1 & 1 & 1 \\
\hline$H$ & & & & & & & 0.346 & 0.346 & 0.346 & 0.491 & 0.491 & 0.491 & 0.552 \\
\hline
\end{tabular}

Notes: the first part of the table enumerates all feasible deprivation bundles in 5 dimensions, and $\pi$ indicates the population share matching each bundle (based on Mozambican data); the second part of the table lists the vectors of true points associated with different switching functions; in columns 1(a) - 2(c) they are the threshold functions described in Table 1, column 3 is the non-threshold function in Eq. (2); $H$ is the poverty headcount, given by the inner product of the population shares and the true points

Source: own calculations 
rows 2(a)-2(c). For each of these functions, I verify whether each unique row-combination of deprivations identifies a unit as poor - i.e., if the weighted sum of deprivations exceeds the cut-off. This exercise, which exhausts all possible combinations of deprivations, confirms that rows 1(a)-1(c) classify all combinations in exactly the same way, as do rows 2(a)-2(c). So, it is no surprise that the overall poverty headcounts reported in the table are identical. The same idea is illustrated visually in Supplementary Figures A1 and A2, which plot the cumulative sum of weights, ordered smallest to largest and vice versa, for each of the identification functions shown in Rows 1 and 2 of Table 1 respectively. In Figure A1, for instance, four steps (weights) are always required either to exceed or to equal the cut-off, indicated by the dashed horizontal line. It is precisely because poverty identification can be described via a (non-smooth) step function that there is a scope for alternative values of $(\vec{w}, k)$ to yield equivalent identification conditions. And here there is non-trivial variation in the weights ascribed to each indicator (and the cut-off), suggested by the coefficient of variation (c.v.) for each column.

The non-uniqueness of weights and cut-offs in terms of poverty identification raises a substantive challenge for the literature that treats differences in the numerical values of these input parameters as reliable and direct indications of differences in who is identified as poor, as well as the relative importance of each indicator. This view is exemplified in debates over how identification functions should be constructed, which focus on choosing specific numerical values for the weights and cut-off (e.g., Alkire 2011b; Alkire et al. 2011; Ravallion 2012; Santos and Villatoro 2018; Abdu and Delamonica 2018; Mitra 2018). Here, the assumption is that variations in the weights and/or cut-off map in a transparent fashion to differences in who is identified as poor. ${ }^{10}$ The same weights are also frequently interpreted as capturing the relative importance of each deprivation indicator. As Alkire and Santos (2014) state: "the weighting structure determines the assumed trade-offs across deprivations" (p. 256; also see Alkire and Foster 2011a). Similarly, in an application of the Alkire-Foster approach, Pasha (2017) notes that: "[w]eights for any composite index of well-being can be based on the trade-offs they imply between the dimensions of well-being." (p. 270). With a similar motivation in mind, Decancq and Lugo (2013) provide a detailed survey of different approaches to setting weights and their underlying rationales, also concluding that weights are crucial factors that determine the trade-off between indicators.

A second concern is that while a given combination of weights and cut-offs may not be unique from the point of view of poverty identification, the adjusted poverty headcount nonetheless remains sensitive to the specific set of weights selected. This is evident from Table 1, where the values for $M_{0}$ are not identical across the various rows. Under the Alkire-Foster procedure, this means that a unique poverty identification function generated from different weights/cut-offs, may be simultaneously associated with a single headcount value but various values of the adjusted headcount (when applied to the same sample data). Put another way, differences in weights such as in Table 1 may have no implications for who is identified as poor, thereby suggesting the numerical differences are arbitrary, but nonetheless have material implications for the estimated poverty intensity. At least from a theoretical viewpoint, this does not seem entirely satisfactory.

\footnotetext{
${ }^{10}$ Similarly, the same inputs are often thought to be appropriate objects for participatory determination and public debate (e.g., Sen and Anand 1997).
} 


\subsection{Functional restrictions}

A second limitation relating to the reliance on weights and a cut-off is that they only admit specific types of identification schemes. As Permanyer (2019) elaborates in detail, only the total amount (weighted sum) of deprivations is taken into account to determine whether a unit is poor, which implicitly makes strong assumptions regarding the degree of substitutability (complementarity) across deprivations. Following the literature, any two deprivations (or groups of deprivations) are said to be substitutes in the production of multidimensional poverty if an increase in any one is sufficient to augment poverty. Similarly, any two deprivations are complements when only their joint increase is sufficient to increase poverty. Under the Alkire-Foster procedure, it is well understood that extreme choices for the cut-off, namely $k \approx 0$ or $k=1$, respectively treat all deprivation indicators as perfect substitutes or complements in the production of multidimensional poverty (see Rippin 2017).

For intermediate choices of $k$, however, the exact nature or degree of substitutability across deprivations is not quite so obvious. To clarify this, denote $w_{(1)}, w_{(2)}, \ldots, w_{(m)}$ as the chosen Alkire-Foster weights for a given function, ordered from smallest to largest. Thus, when $0<k \leq w_{(1)}$ we have $\forall j: w_{j} \geq k$; when $k \leq w_{(1)}+w_{(2)}$ we have $\forall(j, l)$ : $w_{j}+w_{l} \geq k$, and so on. So, for any choice of $k$, we can identify the smallest number of deprivations $p \leq m$, the weighted sum of which will always define a unit as poor (breach the cut-off) and this will hold regardless of which $p$ deprivations are included in the summation. For instance, if $k \leq w_{(1)}$ we have $p=1$; when $k \leq w_{(1)}+w_{(2)} \Leftrightarrow p=2$; when $k=1-w_{(m)} \Leftrightarrow p=m-1$; and when $k=1 \Leftrightarrow p=m$. It follows that a given choice of weights and cut-off defines a $p$-tuple of deprivations, meaning that the intersection of any subset of $p$ deprivations is always sufficient (but not always necessary) to define a unit as poor. And since any one of these $p$-tuples is able to switch the threshold function from zero to one, then any subset of $p$ intersecting deprivations constitutes a perfect substitute for any other. ${ }^{11}$ In other words, given some choice for $(\vec{w}, k)$, the Alkire-Foster approach assumes perfect substitutability between all subsets of intersecting deprivations of at most size $p .^{12}$

The practical limitation here is that groups of deprivations of size $p$ are treated identically for the purpose of identification. This means we generally cannot encode functions where the degree of substitutability varies between distinct sub-groups of deprivation indicators. To see this, imagine that the (earlier) five indicators can be grouped into two mutually exclusive broad poverty dimensions, where the first two indicators capture living conditions and the remaining three capture economic wealth. This is shown in Table 3, which also proposes indicator-specific weights, based on the assumption that each of the two dimensions is equally important. Plausibly, while the different indicators within each dimension might be considered mutual substitutes (i.e., if any single indicator takes a value of one, the household is considered poor in that dimension), the dimensions themselves may be complements in the production of multidimensional deprivation (i.e., the household must be poor in both dimensions simultaneously to be considered multidimensionally poor). However, an additive identification procedure is not able to reflect more complex relationships

\footnotetext{
11 This insight prefigures results to come. Concretely, $p$ is the size of the longest minimal deprivation bundle as defined by a given threshold function. The existence of $p$ also connects to the separability of threshold functions (see below; also de la Vega and Urrutia 2011; Anderson 2019).

12 Alternatively, following Rippin (2017) and taking the perspective of the attributes of well-being (here, $\left.1-d_{i j}\right)$, all corresponding feasible unions of $p$ positive attainments would be perfect complements in the production of multidimensional well-being.
} 
Table 3 Classification of five deprivation indicators into two dimensions

\begin{tabular}{|c|c|c|c|}
\hline \multicolumn{2}{|c|}{ Deprivation indicator } & \multirow{3}{*}{$\begin{array}{l}\text { Dimension } \\
\text { \{ Living conditions }\end{array}$} & \multirow{2}{*}{$\frac{\text { Weight }}{1 / 4}$} \\
\hline$d_{1}$ & Inadequate sanitation & & \\
\hline$d_{2}$ & Inadequate housing materials & & $1 / 4$ \\
\hline$d_{3}$ & No means of transport & \multirow{3}{*}{ Economic wealth } & $1 / 6$ \\
\hline$d_{4}$ & No phone, radio or TV & & $1 / 6$ \\
\hline$d_{5}$ & No fridge, iron or bed & & $1 / 6$ \\
\hline
\end{tabular}

between distinct dimensions of this type. For instance, the intuitive poverty definition given by at least 'one from each dimension' - meaning that to be considered multidimensionally poor, the household must be deprived in at least one indicator within each of the two broad dimensions - cannot be encoded by a single set of weights and a cut-off. To see this, note that while the cut-off $k=\frac{1}{4}+\frac{1}{6}=\frac{5}{12}$ certainly would correctly identify as poor those units deprived in at least one indicator from both dimensions, it would also identify as poor those units deprived in all indicators of one dimension but none of the other. Also, while $k>1 / 2$ would always require units to be deprived in indicators spanning both dimensions, this cut-off requires units to be deprived in at least three indicators to be considered poor. Overall, this clarifies that since only the weights matter under the additive structure of the Alkire-Foster identification scheme, it is not sufficiently flexible to precisely encode more complex poverty definitions, such as 'one from each dimension'.

The restrictive property of threshold functions has been recognised in other contexts. Indeed, the Alkire-Foster poverty identification procedure is equivalent to a weighted voting game, which is just one of various classes of simple yes/no voting games. A yes/no voting game involves several players who vote for or against a proposal; and a weighted game is one where each player has a fixed voting weight and a defined quota must be surpassed to approve a motion (see Taylor and Zwicker 1992; de Keijzer et al. 2012). In our case, the 'players' are the deprivation indicators, 'votes' are binary deprivation values, and the proposal is whether a specific combination of realized deprivations defines a unit as poor. In simple yes/no games, collective approval or disapproval is determined by a decision rule, modelled as: $G=(M ; \mathcal{W})$, where $M$ is the finite set of players (deprivation indicators) and $\mathcal{W}$ is a monotonic set of winning coalitions. A winning coalition represents a particular combination of players (realized deprivations) deemed sufficient to approve a motion (define a unit as poor). Winning coalitions are monotonic in the sense that once a winning coalition contains sufficient positive-voting players, adding more players can never reverse the decision - i.e., for two coalitions (vectors) $x$ and $y$, if $x \in \mathcal{W}$ and $x \subseteq y$ then $y \in \mathcal{W}$.

The above is important since contributions to the literature on monotonic voting games have shown that only certain, arguably restrictive, collective decision rules can be modelled as weighted games. Indeed, the conditions under which a game can be represented as a weighted game are fairly well-known, such as displaying trade-robustness. ${ }^{13}$ Or, as Houy and Zwicker (2014) show, a simple game is weighted if and only if the winning and losing coalitions can be strictly separated by a hyperplane (which corresponds to the choice of cut-off), reflecting the restrictive nature of the decision structure. Viewed in this way, it

\footnotetext{
13 This holds when, for any trade of votes amongst any collection of winning coalitions, at least one of the resulting coalitions continues to be winning.
} 
is evident the Alkire-Foster approach rules-out those poverty identification schemes that are not isomorphic to weighted voting games - i.e., not all identification schemes can be encoded using such functions.

\section{Identification via deprivation bundles}

\subsection{Overview}

In light of these limitations, this section shows there are no major theoretical or practical difficulties in incorporating any valid decision rule (monotonic game or positive switching function), into the framework of multidimensional poverty identification as well as subsequent analytics (e.g., aggregation, adjustment for intensity, decompositions, etc.). The reason for this is straightforward. As previously indicated, by definition, any monotonic game partitions each feasible coalition of players into either the set of winning coalitions or set of losing coalitions. Following Crama and Hammer (2011), who demonstrate that any monotonic game can be represented as a positive switching or Boolean function, a game can be thought of as a switching function that maps any given coalition of positively-voting players to a binary outcome (winning/losing).

The advantage of conceiving of poverty identification in logical (Boolean) terms is that the analysis of (positive) switching functions, which include but are not restricted to threshold functions, focuses on its associated vector of true points. These points index which of the set of all unique combinations of inputs (coalition of players) is winning or losing, as per a truth table. And, critically, this classification of true points is unique to each function. ${ }^{14}$ Thus, for a given positive switching function, $f$, I denote $\mathcal{T}(f)$ as the collection of all unique combinations of inputs for which $f$ is true (non-zero). So, just as Eq. (1) classifies any given combination of deprivations into poor/non-poor categories based on $(\vec{w}, k)$, the truth table associated with any switching function can be used as a fundamental and quite general basis for poverty identification. In the language of multidimensional poverty, each non-empty coalition or input vector can be described as a bundle of deprivations; and the vector of true points associated with the chosen identification function indexes which of these bundles defines a unit as poor.

To see how this works in practice, the columns of Table 2 associated with the identification functions defined in Table 1 list each functions' vector of true points, which are identical for functions 1(a)-(c) and 2(a)-(c), as noted previously. The final column, denoted 3 , provides the true points of a non-threshold function that represents the 'one from each dimension' decision rule described earlier (Table 3). Omitting $i$ subscripts for simplicity this is represented compactly as:

$$
f_{3}=1\left[\left(d_{1}+d_{2}\right) \cdot\left(d_{3}+d_{4}+d_{5}\right) \geq 1\right]
$$

In turn, the poverty headcount associated with any switching function is just the inner product of its vector of true points and the corresponding vector of population/sample shares,

\footnotetext{
14 These are standard results in the analysis of Boolean functions (e.g., for reference see Crama and Hammer 2011). The complete collection of feasible coalitions in $m$ binary indicators is always finite, given by the $m$-fold cartesian product $\mathcal{B}^{m}=\{0,1\}^{m}$.
} 
which give the proportion $(\pi)$ of the population/sample of interest with deprivations exactly matching each bundle. That is:

$$
\begin{aligned}
H(D ; f) & =\sum_{x \in \mathcal{B}^{m}} f(x) \pi(D ; x) \\
& =\sum_{x \in \mathcal{T}(f)} \pi(D ; x)
\end{aligned}
$$

where, as before, $D$ is the observed matrix of deprivations, which can be collapsed into at most $2^{m}$ unique bundles, each denoted $x ; f(x)$ gives the binary output of the chosen identification function for input bundle $x ; \pi(D ; x)$ denotes the population share associated with the same bundle, with property $\sum_{x \in \mathcal{B}^{m}} \pi(D ; x)=1$; and $\mathcal{T}(f)$ is thus the subset of all feasible input combinations for which $f$ is positive (as above). Sub-group decomposability of this measure naturally follows without need for elaboration.

The approach described above advocates using the lens of deprivation bundles as a quite general basis for multidimensional poverty identification. As Eq. (3b) indicates, this places no restrictions on the class of positive switching (Boolean) functions that may be chosen to map deprivation bundles to poor or non-poor categories. By way of a summary, the approach entails five general steps:

1. choose a set of $m$ deprivation indicators (perhaps grouped into broader dimensions);

2. collapse the observed population/sample deprivation data, $D$, into the collection of at most $2^{m}$ unique deprivation bundles (by subgroup), collecting sample or frequency weights as desired;

3. select an identification scheme, $f$ (namely, a positive Boolean function or monotonic game), which maps any set of $m$ binary deprivation indicators onto a binary outcome (poor/non-poor);

4. identify all true points of $f$ (describing which of all feasible bundles define a unit as poor); and

5. calculate the multidimensional poverty headcount as per Eq. (3b).

\subsection{Representation}

Despite the apparent simplicity of the bundles-based approach, the reader might doubt its practicality. One concern is how different functions can be expressed, as well as what kinds of functions are admissible. With respect to the latter, it merits emphasis that a primary advantage of the approach is that a wide range of switching functions can be applied, as long as they are positive monotonic - i.e., for any two bundles $x$ and $y$, it must be that $f(x) \geq f(y)$ if each element of $x$ is greater than or equal to the corresponding element of $y$ $(x \geq y)$. This allows for great flexibility in the kinds of functions that may be incorporated. Furthermore, while there is no single generic generating function that subsumes all positive Boolean functions as special cases, it is nonetheless known that any positive Boolean function (monotonic voting game) can be expressed as the intersection of $c \geq 1$ threshold functions (weighted voting games) (Taylor and Zwicker 1992; Crama and Hammer 2011). While further exploration of this topic extends beyond the scope of this study, suffice to say that $c$ need not be large. For example, the function $f_{3}$ was represented earlier by the intersection of two sub-functions, one for each dimension.

Another complete general representation of any positive Boolean function comes via the the truth table - namely, the set of bundles that classify a unit as poor, hereafter called the set of positive (deprivation) bundles. While this list may be cumbersome when $m$ is large, it can 
be further simplified to focus on the minimal positive bundles (called 'winning coalitions' in voting games or 'prime implicants' of Boolean functions; for further discussion and proof see Crama and Hammer 2011). Any single minimal deprivation bundle can be defined as an intersection of deprivations (indicators) that are both necessary and minimally-sufficient to classify a unit as poor. The minimal sufficiency criterion says that if any indicator included in the bundle were to be set to zero, then the bundle would no longer classify a unit as poor - i.e., all deprivations are 'swing' in a minimal bundle. Thus a minimal deprivation bundle is both: (i) a member of the set of all positive bundles that classify a unit as poor; and (ii) is not absorbed by (is a superset of) any other positive bundle. The benefit of this representation is that the underlying Boolean function can be expressed as the union of all minimal deprivation bundles. So, referring back to the discussion of Section 2, each minimal bundle is a perfect substitute to any other in the production of multidimensional poverty. However, although for threshold functions (e.g., $f_{1}, f_{2}$ ) all bundles equal in length to the longest minimal positive bundle are perfect substitutes (separately define a unit as poor), this does not necessarily hold for other switching functions (e.g., as shown below, the bundle $d_{1} \cdot d_{2}$ is excluded from the minimal bundles of $f_{3}$ ).

To illustrate the unique characterization of identification functions via the set of all minimal bundles, recall the examples of Table 1. According to rows 1(a)-1(c), a unit is classified as poor if they are deprived in any combination of four indicators. Thus, the binary identification function can be represented as the union (disjunction) of all minimal bundles, each of which here contains just four elements and where only the positive elements of each bundle need be included: ${ }^{15}$

$$
\begin{aligned}
f_{1}= & 1\left[\left(d_{1} \cdot d_{2} \cdot d_{3} \cdot d_{4}\right)+\left(d_{1} \cdot d_{2} \cdot d_{3} \cdot d_{5}\right)\right. \\
& \left.+\left(d_{1} \cdot d_{2} \cdot d_{4} \cdot d_{5}\right)+\left(d_{2} \cdot d_{3} \cdot d_{4} \cdot d_{5}\right) \geq 1\right]
\end{aligned}
$$

The function in rows 2(a)-(c) of Table 1 can be uniquely characterised as:

$$
f_{2}=1\left[\left(d_{1} \cdot d_{2} \cdot d_{4} \cdot d_{5}\right)+\left(d_{1} \cdot d_{3}\right)+\left(d_{4} \cdot d_{3}\right)+\left(d_{5} \cdot d_{3}\right) \geq 1\right]
$$

which contains minimal bundles of either two or four elements (dimensions). And the 'one from each dimension' function is uniquely characterized in the normal disjunctive form as:

$$
f_{3}=1\left[\left(d_{1} \cdot d_{3}\right)+\left(d_{2} \cdot d_{3}\right)+\left(d_{1} \cdot d_{4}\right)+\left(d_{2} \cdot d_{4}\right)+\left(d_{1} \cdot d_{5}\right)+\left(d_{2} \cdot d_{5}\right) \geq 1\right]
$$

which is just the long-form of the multiplicative expression used earlier.

In sum, the union of all minimal bundles provides a complete and general way to represent any poverty identification scheme (poverty definition), regardless of its complexity, and from which the vector of true points can be generated. And although such minimal bundles are useful computational tools, it is important to highlight they are not the only valid representational language. Other expressions for Boolean functions - including threshold switching functions - constitute useful simplifications of the disjunctive normal form, but provide the same function. Threshold functions are indeed straightforward to express, especially where the number of indicators is large, but shorter expressions often can be found for non-threshold functions, as shown by Eq. (2) for $f_{3}$.

\footnotetext{
15 By definition, as the function is positive, the zero elements can be excluded. This expression uses the sum-of-products form which, strictly speaking, can be written in modulus 2 arithmetic to dispense with the indicator function. Note this expression is known as the complete disjunctive normal form (DNF) or Blake canonical form of a Boolean function.
} 


\subsection{Intensity}

The material presented to now extends the type of identification functions that might be employed in the analysis of multidimensional poverty, going beyond the threshold functions exclusively admitted under the Alkire-Foster procedure. However, applications of this same procedure rarely focus on the headcount alone - poverty identification constitutes just one component of a multidimensional poverty analysis. The adjusted headcount (see Section 2) is widely used, precisely because it takes into account the intensity of poverty as captured by the weighted sum of deprivations at the unit-level. To incorporate this kind of analysis within the more general framework proposed herein, a counterpart to the deprivation weights is required. The naïve choice would be to apply equal weights, meaning a unit's intensity of deprivation is the sum of deprivations in which she is deprived divided by $m$. This choice is simple and transparent; but equal weights would not capture any potential differences in the relative importance or influence of specific deprivations in the identification of who is poor under different functions.

Drawing on results from monotone (simple) voting games, an attractive option is the normalized Banzhaf index (Dubey and Shapley 1979), which is widely employed to indicate the influence of each voter under a particular set of decision-making rules. ${ }^{16}$ In our context, the corresponding notion of power is the extent to which any single realized deprivation indicator (vote) is critical for whether a unit is classified as poor (the result) - e.g., if across all bundles of deprivations, an indicator is never critical to determine whether that bundle classifies a unit as poor, it would have zero power. The normalized Banzhaf power of each indicator (voter) is calculated as the share of all positive deprivation bundles containing that indicator in which it is a 'swing' (or pivot), which is equal to the number of minimal bundles in which a deprivation indicator appears, divided by the total number of indicators appearing across all minimal bundles. Referring to Eq. (6), for example, we have six minimal bundles containing a total of 12 deprivation indicators. Among these, $d_{1}$ appears three times, implying it takes a Banzhaf power of 3/12 =1/4 (see Table 4). Intuitively, deprivation indicators that appear in more minimal bundles than others will receive a higher power, corresponding to their greater potential influence in determining when a unit is poor at the margin.

Linking to the previous subsection, since this power metric is derived from the set of all minimal bundles used to express each function, it follows that each unique identification function has a single corresponding Banzhaf index. So, even if a threshold Boolean function is employed to generate the minimal bundles (and vector of true points), the Banzhaf index will nonetheless depend exclusively on the structure of these bundles, which is unique to the identification function. This contrasts with the use of numerical weights, which I demonstrated are not necessarily unique to a given function. This implies the Banzhaf index provides a robust and stable metric of influence, varying only with the minimal bundles of each function.

For illustration, Table 4 returns to the previous generating functions and compares the weights used in the Alkire-Foster procedure against the Banzhaf power index (BZ), calculated from the minimal deprivation bundles defined by the same functions. The table also reports the values of a modified Banzhaf index (mBZ), which is calculated in the same fashion as the standard Banzhaf index but broadens the scope to encompass all positive deprivation bundles, not just the set of minimal bundles. This tends to reduce dispersion

\footnotetext{
16 Other, generally more complex, power metrics might also be considered. For further details see Lucas (1983) and Freixas and Gambarelli (1997).
} 
Table 4 Metrics of influence for alternative identification functions

\begin{tabular}{|c|c|c|c|c|c|c|c|c|}
\hline & \multicolumn{3}{|l|}{$f_{1}$} & \multicolumn{3}{|l|}{$f_{2}$} & \multicolumn{2}{|l|}{$f_{3}$} \\
\hline & $\vec{w}$ & $\mathrm{BZ}$ & $\mathrm{mBZ}$ & $\vec{w}$ & $\mathrm{BZ}$ & $\mathrm{mBZ}$ & BZ & $\mathrm{mBZ}$ \\
\hline$d_{1}$ & 0.200 & 0.200 & 0.200 & 0.328 & 0.200 & 0.184 & 0.250 & 0.219 \\
\hline$d_{2}$ & 0.200 & 0.200 & 0.200 & 0.047 & 0.100 & 0.163 & 0.250 & 0.219 \\
\hline$d_{3}$ & 0.200 & 0.200 & 0.200 & 0.459 & 0.300 & 0.286 & 0.167 & 0.188 \\
\hline$d_{4}$ & 0.200 & 0.200 & 0.200 & 0.068 & 0.200 & 0.184 & 0.167 & 0.188 \\
\hline$d_{5}$ & 0.200 & 0.200 & 0.200 & 0.099 & 0.200 & 0.184 & 0.167 & 0.188 \\
\hline$M_{0}$ & 0.307 & 0.307 & 0.307 & 0.422 & 0.388 & 0.387 & 0.426 & 0.420 \\
\hline
\end{tabular}

Note: columns report the influence metrics for different switching functions $\left(f_{1}, f_{2}, f_{3}\right)$ against each deprivation $\left(d_{1}, \ldots, d_{5}\right) ; \vec{w}$ are weights used in the threshold functions; BZ is the normalized Banzhaf power index; and $\mathrm{mBZ}$ is the modified Banzhaf power index; $M_{0}$ is the corresponding adjusted poverty headcount calculated from applying these functions and influence metrics to Mozambican data

Source: own calculations

across the values, which may be helpful where a small number of indicators tend to dominate. The final row of the table gives the corresponding adjusted headcount, calculated from the same Mozambican data reported in Table 2, which is calculated from the normalized sum of deprivations, as described in Section 2, where the normalization uses either the vector of weights or the Banzhaf power index. Three points merit note. First, it is comforting that the three influence metrics are equivalent for the equal weight vector, reflecting the symmetric nature of the identification function. Second, where the weights are unequal, the Banzhaf power index nonetheless clearly allocates the same value to indicators with the same effective influence in the identification process, reflecting the structure of the minimal bundles. Third, even where we do not have weights to enter a threshold function, as in $f_{3}$, the (modified) Banzhaf power index captures meaningful differences in the relative importance of the indicators. For instance, the BZ values here clearly attribute equal weight to each of the two postulated dimensions (1/2 each), as desired.

Finally, combining the identification procedure with metrics of power (or influence) for each deprivation naturally allows for a range of additional analyses in the spirit of AlkireFoster (and related literature), such as decompositions of the contribution of each indicator to either $H$ or $M_{0}$. Taking the latter case, for instance, the decomposition can be written in matrix notation as:

$$
\vec{\pi}\left(D ; \mathcal{B}^{m}\right) \times \operatorname{vecdiag}\left[\vec{f}\left(\mathcal{B}^{m}\right)\right] \times \mathcal{B}^{m} \times \operatorname{vecdiag}[\operatorname{BZ}(f)]
$$

where $\vec{\pi}\left(D ; \mathcal{B}^{m}\right)$ is the vector of observed population/sample shares corresponding to the complete enumeration of bundles (rows of the truth table), $\mathcal{B}^{m}=\{0,1\}^{m} ; \vec{f}\left(\mathcal{B}^{m}\right)$ is the associated vector of true points defined by $f$; vecdiag $[z]$, in an abuse of notation, is the square matrix with vector $z$ on the diagonal and zeros elsewhere; and $\mathrm{BZ}(f)$ gives the power index for function $f$ (also a vector). Reading left to right, the first two terms distribute the population shares over the true points of the function, these are then aggregated across the $m$ deprivation columns and, finally, weighted by the power index. I demonstrate this procedure in the empirical application that follows. 


\section{Application to Mozambique}

This section provides a bare-bones demonstration of how the above procedure can be applied in a somewhat more elaborate and, thus, realistic setting. To do so, I draw on deprivation data from Mozambique, already employed above. The country is of interest since it has achieved one of the world's most rapid and sustained rates of per capita economic growth since the end of conflict in 1992; however, recent consumption poverty estimates have raised concerns as to how well aggregate growth has translated into broad-based welfare gains (DNEAP 2010; Arndt et al. 2012; DEEF 2016). Indeed, the latest household survey from 2014/15 indicates that headcount consumption poverty affected $46 \%$ of the population versus $53 \%$ in $2002 .{ }^{17}$ At the same time, the same surveys indicate more consistent gains in non-consumption dimensions, including ownership of assets and access to public goods, such as education services. This motivates a multidimensional analysis.

In order to proceed, it is necessary to select the deprivations of interest. Although this decision can be controversial, feasible choices are often limited by the availability of consistent data over time, as well as the exclusion of highly correlated indicators. The deprivations selected for the present exercise are summarised in Supplementary Table A1, which reports the share of households deprived in each of eight individual deprivation indicators across four existing waves of nationally-representative household survey data collected by the national statistics agency in 1996/97, 2002/03, 2008/09 and 2014/15. The indicators cover three broader dimensions: human capital (literacy of the household head); housing conditions (access to electricity, water and sanitation, and roofing quality); and ownership of assets or economic wealth (transport assets, information and communications assets, and durable goods). For each deprivation indicator, households who are classified as deprived receive a score of one and zero otherwise. ${ }^{18}$

Supplementary Table A1 indicates changes in well-being have been heterogeneous. While we see progress in all indicators over the full 18 year period, the pace of change is inconsistent. This implies that when constructing a multidimensional metric, the relative importance attributed to different indicators (deprivations), or the specific combination of deprivations used to classify households as poor, is likely to matter. With this in mind, I construct estimates of multidimensional poverty based on the same set of eight variables, applying three alternative identification functions chosen for illustrative purposes. The first, denoted $f_{e}$, contains all minimal bundles of seven deprivations and thereby corresponds to a threshold function with a vector of equal weights and cut-off $k=7 / 8$. The second function, denoted $f_{m}$, remains in the Alkire-Foster tradition but mimics the structure of the global multidimensional poverty index (see Alkire and Santos 2014). It attributes each of the three broad dimensions (human capital, housing conditions and economic wealth) an equal weight, while the indicators underlying each dimension are weighted equally amongst themselves; and the chosen cut-off is $k=3 / 4$. Thirdly, I construct another 'one of each dimension' function, which cannot be represented by a single Boolean threshold function. This defines a household as poor if it is deprived in the human capital dimension as well as at least one indicator from each of the other dimensions, as given by: $f_{t}=\left[d_{1} \cdot\left(\sum_{j=2}^{5} d_{j}\right) \cdot\left(\sum_{j=6}^{8} d_{j}\right) \geq 1\right]$, where $\left(d_{1}, d_{2}, \ldots, d_{8}\right)$ refer to the deprivations in the order shown in Supplementary Table A1 (top to bottom).

\footnotetext{
17 Survey weights are applied in all estimates in this section.

18 Further details on the data and construction of the underlying deprivation dimensions is available on request from the author (see also, DEEF 2016). For the exercise in Section 2, the same data from 2014/15 is used, but the first three indicators are excluded.
} 
Table 5 Adjusted poverty headcount decomposition, for alternative identification functions

\begin{tabular}{|c|c|c|c|c|c|c|}
\hline & \multicolumn{2}{|l|}{$f_{e}$} & \multicolumn{2}{|l|}{$f_{m}$} & \multicolumn{2}{|l|}{$f_{t}$} \\
\hline & 1997 & 2014 & 1997 & 2014 & 1997 & 2014 \\
\hline \multicolumn{7}{|c|}{ (a) Vector weight-based decomposition } \\
\hline Literacy & 0.049 & 0.022 & 0.148 & 0.084 & 0.094 & 0.063 \\
\hline Electricity & 0.068 & 0.026 & 0.037 & 0.021 & 0.050 & 0.032 \\
\hline Clean water & 0.063 & 0.022 & 0.031 & 0.015 & 0.042 & 0.022 \\
\hline Sanitation & 0.068 & 0.025 & 0.037 & 0.020 & 0.050 & 0.030 \\
\hline Roofing & 0.067 & 0.025 & 0.034 & 0.019 & 0.046 & 0.028 \\
\hline Transport & 0.066 & 0.023 & 0.045 & 0.023 & 0.049 & 0.029 \\
\hline Information & 0.063 & 0.021 & 0.041 & 0.018 & 0.044 & 0.019 \\
\hline Durables & 0.068 & 0.026 & 0.049 & 0.028 & 0.053 & 0.033 \\
\hline$M_{0}$ & 0.513 & 0.189 & 0.423 & 0.228 & 0.427 & 0.257 \\
\hline$H$ & 0.547 & 0.204 & 0.444 & 0.252 & 0.457 & 0.308 \\
\hline \multicolumn{7}{|c|}{ (b) Banzhaf power-based decomposition } \\
\hline Literacy & 0.049 & 0.022 & 0.077 & 0.044 & 0.152 & 0.103 \\
\hline Electricity & 0.068 & 0.026 & 0.052 & 0.030 & 0.038 & 0.024 \\
\hline Clean water & 0.063 & 0.022 & 0.045 & 0.022 & 0.032 & 0.016 \\
\hline Sanitation & 0.068 & 0.025 & 0.052 & 0.028 & 0.038 & 0.023 \\
\hline Roofing & 0.067 & 0.025 & 0.049 & 0.027 & 0.034 & 0.021 \\
\hline Transport & 0.066 & 0.023 & 0.048 & 0.024 & 0.046 & 0.027 \\
\hline Information & 0.063 & 0.021 & 0.044 & 0.019 & 0.041 & 0.018 \\
\hline Durables & 0.068 & 0.026 & 0.052 & 0.029 & 0.050 & 0.031 \\
\hline$M_{0}$ & 0.513 & 0.189 & 0.418 & 0.223 & 0.431 & 0.264 \\
\hline$H$ & 0.547 & 0.204 & 0.444 & 0.252 & 0.457 & 0.308 \\
\hline
\end{tabular}

Notes: columns give the adjusted poverty headcount decompositions, as well as aggregate adjusted and total poverty headcounts, for different switching functions $f_{e}, f_{m}, f_{t}$ (described in the text); panel (a) applies vector weights in the decomposition procedure (or in the case of $f_{t}$ the modified Banzhaf weight); panel (b) employs the normalised Banzhaf power index associated with each function

Source: own calculations

Using each of these three identification functions, Table 5 reports the headcount, adjusted headcount and decomposition of the adjusted headcount (by indicator). As the latter two depend on the choice of influence metric, I show results based on the vector weights used to calculate the intensity of poverty, given in panel (a) as per the conventional AlkireFoster approach, as well as the Banzhaf power metric, given in panel (b). ${ }^{19}$ Supplementary Figure A3 visualizes the same decompositions for functions $f_{m}$ and $f_{t}$. Two main findings emerge. First, while there are some clear similarities between the weight-based and Banzhaf power-based results, particularly within each function and after aggregation, the decompositions are not alike. For instance, for function $f_{m}$ the contribution of literacy changes by

\footnotetext{
${ }^{19}$ Since function $f_{t}$ does not have an associated vector of weights, for this exercise I use the modified Banzhaf power index.
} 
a factor of two between panels (a) and (b) in both periods. This reflects the point that the chosen weights provide a poor guide to the effective influence of this dimension in the identification function, a point confirmed in Table A2 that shows the Banzhaf power index for each function. Second, and in contrast, the non-threshold function, $f_{t}$, in fact precisely captures the proposed influence of each indicator represented by the vector of weights used in $f_{m}$ (see also Table A2). Here literacy plays a more decisive role and, given the slower progress in this indicator shown in Supplementary Table A1, this explains the slower pace of poverty reduction (on aggregate) over time viewed from this function. Put differently, the less permissive structure of $f_{t}$ compared to $f_{m}$, where the former limits the degree of substitution between indicators from different dimensions, yields a somewhat less rosy view of trends in poverty reduction.

\section{Conclusion}

This paper had two main objectives. The first was to revisit some limitations of the AlkireFoster approach to poverty identification, which relies on a threshold switching function, including the emphasis placed on the numerical values of weights and cut-offs used in this process. I showed that weights and cut-offs are generally not unique to a given function and, thus, weights do not necessarily reflect the relative importance of each deprivation indicator. A further critical limitation is that threshold switching functions are a fairly restrictive class of switching (Boolean) functions, which implicitly assume that all intersecting groups of indicators of a fixed size (as defined by the weights and cut-off) are perfect substitutes in the production of multidimensional poverty. Thus, in treating groups of deprivations of this size as interchangeable, they generally cannot encode certain intuitive identification schemes, such as a 'one from each dimension' rule.

The second objective was to extend the scope of poverty identification to incorporate other types of positive switching functions. To do so, I proposed using the complete set of deprivation bundles and the associated vector of true points, which are unique to each function and provide a natural basis for poverty identification. Furthermore, I showed that the set of minimal positive deprivation bundles can be used as a simple and unique representation of any chosen function, from which metrics of the influence of each deprivation indicator also can be calculated, namely the normalized Banzhaf power index. In this way, adjusted poverty headcounts and further decompositions can be derived, which - unlike numerical weights - directly reflect the true points of the chosen identification function. The application to Mozambique demonstrated the straight-forward and practical nature of the proposed procedure. In particular, it showed how a 'one from each dimension' function can be deployed easily, yielding distinct insights that allow for perfect complementarity between aggregate dimensions that cannot be replicated with a threshold switching function.

It is important to highlight that the procedure outlined here represents an extension of the Alkire-Foster approach to a wider range of switching functions. In many cases, a threshold function can be perfectly adequate to express the poverty definition of interest. Nonetheless, as I have argued, one must be careful not to conflate the specific numerical weights in the threshold function with the relative importance of each indicator. So, even in these cases, the more general procedure I have proposed is helpful since it assures poverty intensities reflect the unique structure of the identification function. Also, while the deployment of other (nonthreshold) switching functions can entail greater complexity, this is not necessarily the case even with large numbers of indicators. Indeed, 'one from each dimension' type functions are neither difficult to represent compactly nor difficult to encode computationally. And, 
in principle, any switching function can be constructed from the intersection of multiple threshold sub-functions.

Finally, this procedure hints at possible areas for future research. The representation of identification functions using minimal bundles suggests these bundles could become a fertile basis for the (participatory) construction of poverty definitions, including the use of natural language expressions to arrive at generating functions. Also, further applied work using non-threshold functions is likely to be of value to track progress over time under the assumption that different dimensions are strict complements (substitutes) in the production of multidimensional poverty (well-being).

Supplementary Information The online version contains supplementary material available at (https://doi. org/10.1007/s10888-021-09477-8).

Acknowledgements Thanks to the editor, referees, Paul Anand, Paola Ballon, James Foster, Simon Quinn, Vincenzo Salvucci, Ricardo Santos, Suman Seth, Finn Tarp, Erik Thorbecke and Gaston Yalonetzky for comments and encouragement on my investigation of this topic. This is a revised and updated version of a study originally commissioned by UNU-WIDER (Helsinki), under the project 'Inclusive Growth in Mozambique: scaling-up research and capacity'. All errors and omissions are my own.

Open Access This article is licensed under a Creative Commons Attribution 4.0 International License, which permits use, sharing, adaptation, distribution and reproduction in any medium or format, as long as you give appropriate credit to the original author(s) and the source, provide a link to the Creative Commons licence, and indicate if changes were made. The images or other third party material in this article are included in the article's Creative Commons licence, unless indicated otherwise in a credit line to the material. If material is not included in the article's Creative Commons licence and your intended use is not permitted by statutory regulation or exceeds the permitted use, you will need to obtain permission directly from the copyright holder. To view a copy of this licence, visit http://creativecommons.org/licenses/by/4.0/.

\section{References}

Abdu, M., Delamonica, E.: Multidimensional child poverty: from complex weighting to simple representation. Soc. Indic. Res. 136(3), 881-905 (2018)

Alkire, S., Foster, J.: Counting and multidimensional poverty measurement. J. Public Econ. 95, 476-487 (2011a)

Alkire, S.: Understandings and misunderstandings of multidimensional poverty measurement. J. Econ. Inequal. 9(2), 289-314 (2011b)

Alkire, S., Foster, J., Santos, M.: Where did identification go? J. Econ. Inequal. 9(3), 501-505 (2011)

Alkire, S., Santos, M.E.: Measuring acute poverty in the developing world: Robustness and scope of the multidimensional poverty index. World Dev. 59, 251-274 (2014)

Alkire, S., Seth, S.: Multidimensional poverty reduction in India between 1999 and 2006: Where and how? World Dev. 72, 93-108 (2015)

Anand, P., Durand, M., Heckman, J.: The measurement of progress - some achievements and challenges. Journal of the Royal Statistical Society: Series A (Statistics in Society) 174(4), 851-855 (2011)

Anderson, G.: Multilateral Wellbeing Comparison in a Many Dimensioned World: Ordering and Ranking Collections of Groups. Palgrave Macmillan, Cham (2019)

Angulo, R., Díaz, Y., Pardo, R.: The Colombian multidimensional poverty index: Measuring poverty in a public policy context. Soc. Indic. Res. 127(1), 1-38 (2016)

Arndt, C., Hussain, M.A., Jones, E.S., Nhate, V., Tarp, F., Thurlow, J.: Explaining the evolution of poverty: the case of Mozambique. American Journal of Agricultural Economics. https://doi.org/10.1093/ajae/aas022 (2012)

Atkinson, A.: Multidimensional deprivation: contrasting social welfare and counting approaches. J. Econ. Inequal. 1(1), 51-65 (2003)

Bennett, C., Mitra, S.: Multidimensional poverty: measurement, estimation and inference. Econ. Rev. 32(1), 57-83 (2013) 
Bourguignon, F., Chakravarty, S.: The measurement of multidimensional poverty. J. Econ. Inequal. 1(1), 25-49 (2003)

Crama, Y., Hammer, P.L.: Boolean Functions: Theory, Algorithms, and Applications. Cambridge University Press, Cambridge (2011)

Decancq, K., Lugo, M.A.: Weights in multidimensional indices of wellbeing: an overview. Econ. Rev. 32(1), 7-34 (2013)

DEEF: Pobreza e bem-estar em Moçambique: Quarta avaliação nacional (IOF 2104/15). Technical report, Direcção de Estudos Económicos e Financeiros, Ministério de Economia e Finanças, República de Moçambique. https://www.wider.unu.edu/sites/default/files/Final_QUARTAAVALIA (2016)

DNEAP: Pobreza e bem-estar em Moçambique: Terceira avaliação nacional. Technical report, Ministry of Planning and Development, Government of Mozambique. www.dneapmpd.gov.mz/index.php? option=com_docman\&task=doc_download\&gid=133\&Itemid=54 (2010)

Dubey, P., Shapley, L.S.: Mathematical properties of the Banzhaf power index. Math. Oper. Res. 4(2), 99-131 (1979)

Foster, J., Greer, J., Thorbecke, E.: A class of decomposable poverty measures. Econometrica 52(3), 761-766 (1984)

Foster, J.E., McGillivray, M., Seth, S.: Composite indices: rank robustness, statistical association, and redundancy. Econom. Rev. 32(1), 35-56 (2013)

Freixas, J., Gambarelli, G.: Common internal properties among power indices. Control. Cybern. 26, 591-604 (1997)

Houy, N., Zwicker, W.S.: The geometry of voting power: weighted voting and hyper-ellipsoids. Games and Economic Behavior 84, 7-16 (2014)

Jones, S.: Measuring what's missing: practical estimates of coverage for stochastic simulations. J. Stat. Comput. Simul. 86(9), 1660-1672 (2016)

de Keijzer, B., Klos, T.B., Zhang, Y.: Solving weighted voting game design problems optimally: Representations, synthesis, and enumeration. ERIM Report Series Reference No. ERS-2012-006-LIS, Erasmus Research Institute of Management. arXiv:1204.5213 (2012)

Lucas, W.F.: Measuring power in weighted voting systems. In: Brams, S.J., Lucas, W.F., Straffin, P.D. (eds.) Political and Related Models, pp. 183-238. Springer, New York (1983)

Maasoumi, E.: The measurement and decomposition of multi-dimensional inequality. Econometrica 54, 991997 (1986)

Mitra, S.: Re-assessing "trickle-down" using a multidimensional criteria: the case of India. Soc. Indic. Res. 136(2), 497-515 (2018)

Pasha, A.: Regional perspectives on the multidimensional poverty index. World Dev. 94, 268-285 (2017)

Permanyer, I.: Measuring poverty in multidimensional contexts. Soc. Choice Welf. 53(4), 677-708 (2019)

Ravallion, M.: Mashup indices of development. The World Bank Research Observer 27(1), 1-32 (2012)

Rippin, N.: Efficiency and distributive justice in multidimensional poverty issues. In: White, R. (ed.) Measuring Multidimensional Poverty and Deprivation: Incidence and Determinants in Developed Countries, chapter 3, pp. 31-67. Springer International Publishing (2017)

Santos, M.E., Villatoro, P.: A multidimensional poverty index for Latin America. Rev. Income Wealth 64(1), 52-82 (2018)

Sen, A., Anand, S.: Concepts of Human Development and poverty: A Multidimensional perspective, pp. 120. New York: United Nations Development Programme. Reprinted in S. Fukuda-Parr and A. K. Shiva Kumar, eds. Readings in Human Development (New Delhi: Oxford University Press, 2003) (1997)

Seth, S.: Inequality, interactions, and human development. J. Hum. Dev. Capab. 10(3), 375-396 (2009)

Seth, S., Alkire, S.: Did poverty reduction reach the poorest of the poor? Complementary measures of poverty and inequality in the counting approach. Research on Economic Inequality 25, 63-102 (2017)

Taylor, A., Zwicker, W.: A characterization of weighted voting. Proc. Am. Math. Soc. 115(4), 1089-1094 (1992)

de la Vega, M.C.L., Urrutia, A.: Characterizing how to aggregate the individuals' deprivations in a multidimensional framework. J. Econ. Inequal. 9(2), 183-194 (2011)

Publisher's note Springer Nature remains neutral with regard to jurisdictional claims in published maps and institutional affiliations. 